\title{
Formation of ultrashort pulses with sub-Poissonian photon statistics
}

\author{
F. Popescu*, A. S. Chirkin ${ }^{\dagger}$ \\ Moscow State University, \\ 119899 Moscow, Russian Federation \\ E-mail: *florentin_p@hotmail.com, ${ }^{\dagger}$ chirkin@foton.ilc.msu.su
}

(March 10, 2000)

A simple method for the production of ultrashort light pulses (USPs) with suppressed photon fluctuations is considered. The method is based on self-phase modulation (SPM) of an USP in a nonlinear medium (optical fibre) and subsequent transmission of pulse through a dispersive optical element.

PACS numbers: 42-50.Dv, 42-81.Dp

Enlarged version of the article published in Quantum Electronics, 29 No. 7, 61-63 (1999)

Ultrashort light pulses (USPs) continue to draw the attention of investigators today. The state of the art in this area of laser physics in the late 1980s was set forth by S. A. Akhmanov et al. in Refs. [1], [2]. In the past decade, considerable progress has been made in quantum optics in the generation of nonclassical (the so called squeezed) light fields. The formation and application of USPs in a nonclassical state makes possible to combine in experiments a high time resolution with a low level of fluctuations.

In principle, one can obtain pulsed light fields in a nonclassical state by using the same nonlinear optical interaction as those used in the case of continuous fields [3], [1]. Parametric amplification is a technique that is most extensively used for this purpose nowadays. In the case of degenerate three-frequency parametric amplification, quadrature-squeezed light is produced. However, this light is found to have super-Poissonian statistics directly at the output of the amplifier, and one needs interferometers to transform it to get sub-Poissonian statistics. One can obtain light with sub-Poissonian photon statistics with the aid of nonlinear interferometric devices in the presence of self-phase modulation (see, e.g., Ref. [3]). Note that the self-phase modulation itself is not accompanied by a change in photon statistics.

In a recent paper, we studied SPM of a light pulse and its subsequent propagation in a dispersive linear medium (or the passage through optical compressors). In this case, pulses with sub-Poissonian photon statistics can be formed. We managed to make an accurate calculation of the process under consideration owing to the consistent quantum theory developed by us for the self-action of light pulses in a medium with inertial nonlinearity [5], [6]. The method of formation of pulses with sub-Poissonian statistics proposed here is simple for experimental realization and stable against external random effects (technical fluctuations). Note that the theory developed in Refs. [5], [6] for the self-effect of USPs takes into account a relaxation time of a nonlinearity that determines the region of the spectrum of quantum fluctuations which are of substantial importance in the formation of squeezed light and does not limit the amplitude of quantum fluctuation.

When analysed from the quantum point of view, SPM of USPs is described by the expression [5], [6].

$$
\hat{A}(t, l)=e^{\hat{O}(t)} \hat{A}_{0}(t),
$$

and the Hermite conjugate operator $\hat{A}^{+}(t, l)$. Here $\hat{A}^{+}(t, l)(\hat{A}(t, l))$ is the photon creation (annihilation) operator for the given section $x$ at a given moment of time $t$ (the output of a nonlinear medium is specified by the section $x=l$, and the input is specified by the section $\left.x=0, \hat{A}(t, x=0)=\hat{A}_{0}(t)\right), \hat{O}(t)=i \gamma q\left[\hat{n}_{0}(t)\right]$, and $\gamma$ is the coefficient related to nonlinear properties of a medium and proportional to the length $l$ in it. The expression $\gamma q\left[\hat{n}_{0}(t)\right]$ characterizes the nonlinear phase incursion,

$$
q\left[\hat{n}_{0}(t)\right]=\int_{-\infty}^{\infty} H\left(\left|t_{1}\right|\right) \hat{n}_{0}\left(t-t_{1}\right) d t_{1},
$$

and $\hat{n}_{0}(t)=\hat{A}_{0}^{+}(t) \hat{A}_{0}(t)$ is the operator of the "density" of the number of photons at the input of a nonlinear medium. The function $H(t)$ takes into account a finite response time of the nonlinearity of a medium. In view of the causality principle, $H(t) \neq 0$ for $t \geq 0$ and $H(t)=0$ for $t<0$. In expression (11) and (2), $t=t^{\prime}-x / u$ is the time in the moving coordinate system, $t^{\prime}$ is the current time, and $u$ is the pulse velocity in a medium.

In the case of SPM (see (11)) discussed here, the quadrature-squeezed light [5], [6] is formed, and the photon statistics in a medium is not changed because the operator of the number of photons $\hat{n}(t, l)=\hat{n}_{0}(t)$ remains unchanged. In what follows, we show that the propagation of a pulse (see (1)) through a dispersive optical device is able to transform its initial Poissonian statistics into sub- or super-Poissonian statistics.

For example, let us consider the propagation of a pulse in a dispersive linear medium. At the output of the 
medium, we have the following expression for the photon annihilation operator [2]:

$$
\hat{B}(t, z)=\int_{-\infty}^{\infty} G\left(t-t_{1}, z\right) \hat{A}\left(t_{1}, l\right) d t_{1} .
$$

It is known that the operators $\hat{A}(t, l)$ and $\hat{B}(t, z)$ are bound to satisfy commutation relation of the $\left[\hat{C}\left(t_{1}\right), \hat{C}^{+}\left(t_{2}\right)\right]=\delta\left(t_{1}-t_{2}\right)$ type. In view of relation (3) between the operators under consideration, we find the condition imposed on the Green function $G(t)$ of the dispersive element:

$$
\int_{-\infty}^{\infty} G\left(t_{1}-t, z\right) G^{*}\left(t_{2}-t, z\right) d t=\delta\left(t_{2}-t_{1}\right) .
$$

Let us introduce the operator of the number of photons over the measurement time $\mathcal{T}$,

$$
\hat{N}_{\mathcal{T}}(t, z)=\int_{t-\mathcal{T} / 2}^{t+\mathcal{T} / 2} \hat{N}\left(t_{1}, z\right) d t_{1},
$$

and define the Mandel parameter

$$
Q(t, z)=\frac{\varepsilon(t, z)}{\left\langle\hat{N}_{\mathcal{T}}(t, z)\right\rangle},
$$

where

$$
\begin{aligned}
\hat{N}(t, z) & =\hat{B}^{+}(t, z) \hat{B}(t, z) \\
\varepsilon(t, z) & =\left\langle\hat{N}_{\mathcal{T}}^{2}(t, z)\right\rangle-\left\langle\hat{N}_{\mathcal{T}}(t, z)\right\rangle^{2}-\left\langle\hat{N}_{\mathcal{T}}(t, z)\right\rangle .
\end{aligned}
$$

The parameter $Q(t, z)$ characterizes the difference between photon statistics and Poissonian statistics (for the latter $Q(t, z)=0)$. The angle brackets in (7) denote averaging over the initial quantum state of the pulse.

One can calculate expression (7) by using the algebra of time dependent Bose operators [5], [6]. In this case we have the following relations:

$$
\begin{gathered}
\hat{A}_{0}\left(t_{1}\right) e^{\hat{O}\left(t_{2}\right)}=e^{\hat{O}\left(t_{2}\right)+\mathcal{H}\left(t_{2}-t_{1}\right)} \hat{A}_{0}\left(t_{1}\right), \\
e^{\hat{O}(t)}=\hat{\mathbf{N}} \exp \left\{\int_{-\infty}^{\infty}\left[e^{\mathcal{H}\left(t_{1}\right)}-1\right] \hat{n}_{0}\left(t-t_{1}\right) d t_{1}\right\},
\end{gathered}
$$

where $\mathcal{H}(t)=i \gamma h(t), h(t)=H(|t|)$ and $\hat{\mathbf{N}}$ is the operator of normal ordering.

In the case of a coherent initial pulse, a small nonlinear phase incursion per photon, and the pulse duration $\tau_{p}$ is considerably grater than the times $\mathcal{T}$ and $\tau_{r}\left(\tau_{r}\right.$ is the nonlinearity relaxation time). After some cumbersome calculations, taking into account expression (8) and (9) one gets

$$
\varepsilon(t, z)=2 \gamma \mathcal{T}^{2} \operatorname{Im}\left[I_{1}^{2}(t, z) I_{2}^{*}(t, z)\right]
$$

where

$$
\begin{aligned}
I_{1}(t, z) & =\int_{-\infty}^{\infty} G\left(t-t_{1}, z\right) \alpha\left(t_{1}\right) e^{i \psi\left(t_{1}\right)} d t_{1} \\
I_{2}(t, z) & =\int_{-\infty}^{\infty} \int_{-\infty}^{\infty} G\left(t-t_{1}, z\right) G\left(t-t_{2}, z\right) \alpha\left(t_{1}\right) \alpha\left(t_{2}\right) \\
& \times h\left(t_{1}-t_{2}\right) e^{i\left[\psi\left(t_{1}\right)+\psi\left(t_{2}\right)\right]} d t_{1} d t_{2}
\end{aligned}
$$

Here $\alpha(t)$ is the eigenvalue of the operator $\hat{A}_{0}(t) ; \psi(t)=$ $2 \gamma \bar{n}_{0}(t)$ is the nonlinear phase incursion, $\bar{n}_{0}(t)=|\alpha(t)|^{2}$ is the average density of the initial number of photons, and $\left\langle\hat{N}_{\mathcal{T}}(t, z)\right\rangle=\mathcal{T}\left|I_{1}(t, z)\right|^{2}$. form

Let us assume that the Green function $\mathrm{G}(\mathrm{t})$ has the

$$
G(t, z)=\left(-i 2 \pi k_{2} z\right)^{-1 / 2} \exp \left(-\frac{i t^{2}}{2 k_{2} z}\right),
$$

where $z$ is the distance traveled in the dispersive linear medium. We have the coefficients $k_{2}>0$ for normal dispersion of the group velocity, and $k_{2}<0$ for anomalous dispersion. Expression (13) corresponds to the case when dispersive properties of a medium are taken into account in the second approximation of the dispersion theory [2].

The dispersion effect is known [7] to have a spatial analogue in the form of diffraction spreading of a wave beam. The quantum description of diffraction in nonlinear optical processes was considered first by S. A. Akhmanov et al. in Ref. [8] (see also Ref. [3]), where it was shown that the mixing of different angular components of a parametrical amplified beam is able to cause additional nonclassical effects. One can expect similar effects in the case under consideration. The self-interaction effect of a pulse given by expression (11) is accompanied by changes in its phase (and therefore frequency) over time. In the course of its passage through dispersive linear elements, a frequency-modulated pulse of this type is compressed or stretched 2. It is precisely this effect that is able to change its photon statistics.

Let us determine the parameter $Q(t, z)$. To simplify calculations, we take the nonlinearity response function in the form $h(t)=\left(1 / \tau_{r}\right) \exp \left(-t^{2} / \tau_{r}^{2}\right)$. Let a pulse be initially of the Gaussian form $\bar{n}_{0}(t)=\bar{n}_{0} \exp \left(-t^{2} / \tau_{p}^{2}\right)$. In the paraxial approximation, i.e., in the case where the phase $\psi(t)$ is replaced with $\psi_{0}\left(1-t^{2} / \tau_{p}^{2}\right),\left(\psi_{0}=2 \gamma \bar{n}_{0}\right)$, we obtain for $\tau_{p}>\tau_{r}$ :

$$
\left\langle\hat{N}_{\mathcal{T}}(t, z)\right\rangle=\bar{n}_{0} \mathcal{T} V^{-1}(z) \exp \left(-\frac{t^{2}}{V^{2}(z) \tau_{p}^{2}}\right),
$$

$Q(0, z)=-\psi_{0}\left[\frac{\mathcal{T}}{\tau_{p}}\right] \sin \left\{\arctan \left(\frac{\varphi(z)}{\varpi(z)}\right)\right.$ 


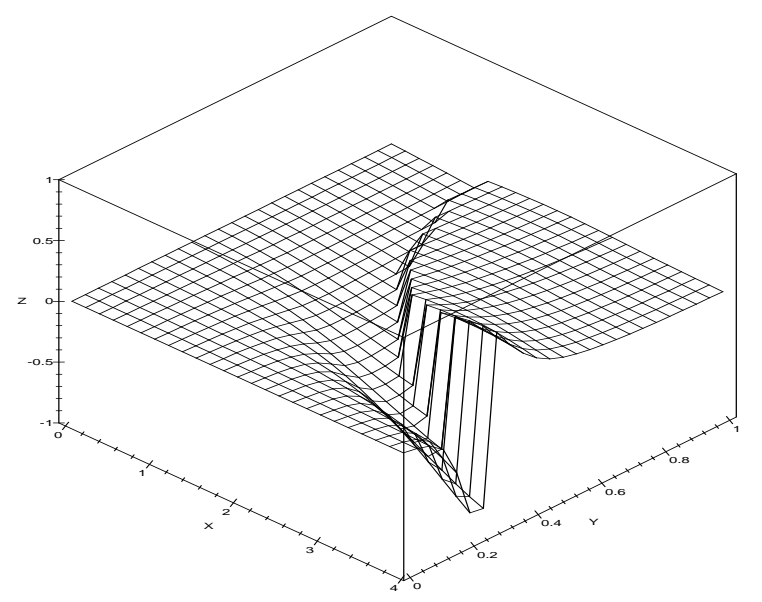

FIG. 1. Dependence of the parameter $Q(0, z)$ [Z] in the case of compression of a USP with SPM on the nonlinear phase incursion $\psi_{0}[\mathrm{X}]$ and the dispersion phase $\varphi(z)[\mathrm{Y}]$ for $\tau_{p} / \mathcal{T}=8$.

$$
\begin{aligned}
& \left.+\frac{1}{2} \arctan \left(\frac{2 \varphi_{d}(z) \varpi(z)}{2 \varphi(z) \varphi_{d}(z)-\varpi^{2}(z)}\right)\right\} \\
& /\left[\varpi^{4}(z)-2 \varphi^{2}(z) \varpi^{2}(z)+4 \varphi^{4}(z)\right]^{1 / 4}
\end{aligned}
$$

where

$$
\begin{aligned}
& V^{2}(z)=\varpi^{2}(z)+\varphi^{2}(z) ; \quad \varpi(z)=1-s \psi_{0} \varphi(z) \\
& \varphi(z)=\frac{z}{D} ; \quad \varphi_{d}(z)=\frac{z}{d} ; \quad D=\frac{\tau_{p}^{2}}{\left|k_{2}\right|} ; \quad d=\frac{\tau_{r}^{2}}{\left|k_{2}\right|}
\end{aligned}
$$

$D$ and $d$ are the characteristic dispersion lengths, $s=1$ for $k_{2}<0$, and $s=-1$ for $k_{2}>0$. Let us restrict our consideration to the analysis of the parameter $Q(t, z)$ for $t=0$, because expression (15) for an arbitrary moment of time $t$ is rather cumbersome.

From (14) and (15) it follows that one can have $Q(t, z)<0$, i.e., obtain a pulse with sub-Poissonian photon statistics through both compression $(s=1)$ and spreading $(s=-1)$ of a pulse. Of particular interest from the practical point of view is the case of anomalous group velocity dispersion $(s=1)$ in which the compression of a phase-modulated pulse (see (14)) takes place. The dependence of the Mandel parameter and the average number of photons in this case are presented in Fig.파.Fig.2 2 and Fig.3. One can see that the suppression of fluctuation of the number of photons becomes noticeable for the nonlinear phase $\psi_{0}>1$. For a given nonlinear phase $\psi_{0}$, there is a certain dispersive phase incursion for which the suppression of the number of photons is the greatest. According to (14), this is obtained for $\varphi_{\text {opt }}(z)=1 / \psi_{0}$. In this case, the Mandel parameter has the minimum value $Q_{\text {min }}=-\left[\mathcal{T} / \tau_{p}\right] \psi_{0}^{2}$. Note that a pulse has the minimal duration for $\varphi(z)=\psi_{0} /\left(1+\psi_{0}^{2}\right)$. For $\psi_{0} \gg 1$ both extremities are almost coincident.

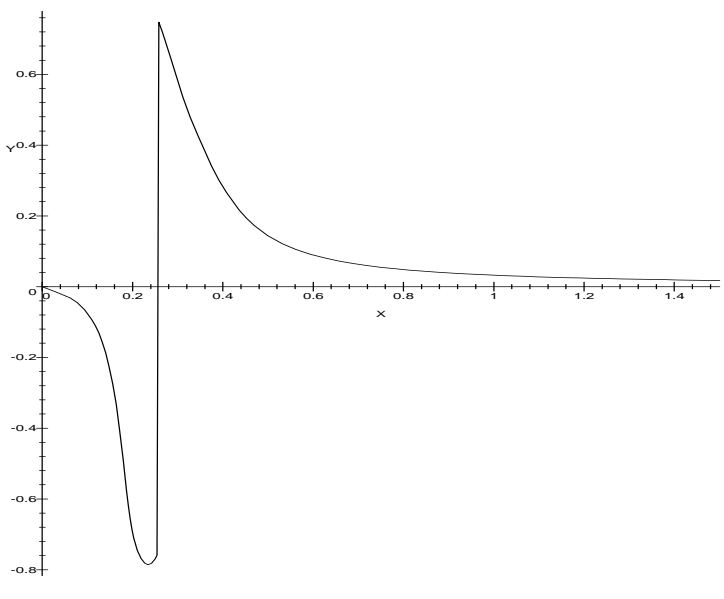

FIG. 2. Dependence of the parameter $Q(0, z)[\mathrm{Y}]$ in the case of compression of a USP with SPM on the dispersion phase $\varphi(z)[\mathrm{X}]$ for $\psi_{0}=4$ and $\tau_{p} / \mathcal{T}=8$.

As follows from calculations, the photon statistics for the measurement time $\mathcal{T} \gg \tau_{p}$ is of the Poissonian type. A situation identical in many aspects to the one considered here was observed in spectral measurements of fluctuations of femtosecond pulses transmitted through an optical fibre in the region of normal group-velocity dispersion. An increase in the spectral bandwidth of a filter caused an increase of the level of fluctuations [9].

Let us present a numerical example. Consider the interaction of a pulse at a wavelength of $1 \mu \mathrm{m}$ in an optical fibre, and let its initial duration and maximum intensity be $2 \tau_{p}=10 \mathrm{ps}$ and $10^{7} \mathrm{~W} \cdot \mathrm{cm}^{-2}$. In this case, the nonlinear phase in a quartz fibre for the path length $l=100 \mathrm{~m}$ is $\psi_{0}=3$. If the resulting USP travels through an optical compressor with anomalous dispersion $\left|k_{2}\right|=$ $10^{-26} \mathrm{~s}^{2} \cdot \mathrm{cm}^{-1}$, the dispersion phase $\varphi_{\text {opt }}(z)=1 / \psi_{0}$ is obtained at a distance of about $8 \mathrm{~m}$. This example illustrates the feasibility of formation of USPs with sub-Poissonian photon statistics by the method proposed above.

The obtained result shows that it is possible in principle to form a pulse with sub-Poissonian photon statistics in the case where a high-intensity pulse undergoes SPM during its travel through a nonlinear medium and subsequently passes a dispersive optical device (an optical compressor or an optical fibre). In view of the fact that these processes occur in succession, one can choose an optimum scheme or optimum conditions at each transformation stage. In the specific case of formation of lowintensity USPs in a nonclassical state, one can attenuate high-intensity pulses at the output of a nonlinear medium. It is natural that this is accompanied by a partial loss of nonclassical properties. It should be noted that in the case of formation of optical solitons, SPM and the dispersion effect take place simultaneously [2].

Authors are grateful to V. A. Vysloukh for fruitful discus- 


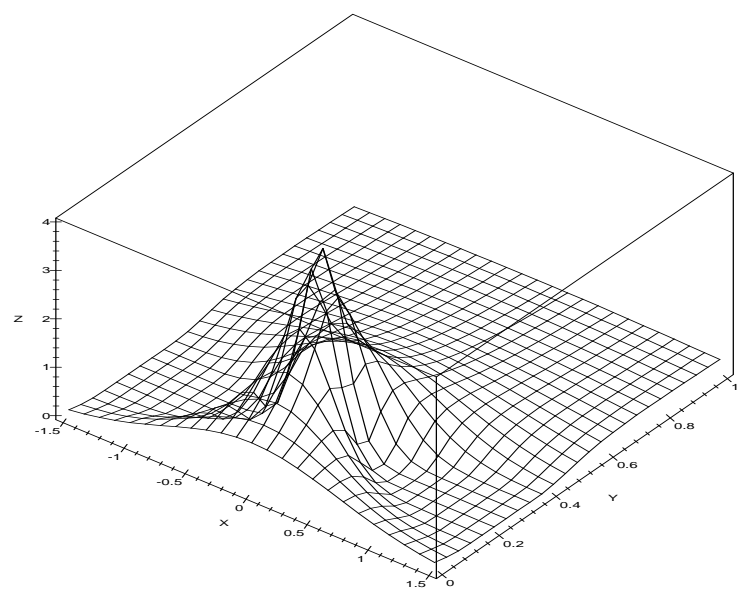

FIG. 3. Variation of the shape of a USP with SPM during compression. The calculation are made in the paraxial approximation. $\left([\mathrm{Z}] \equiv\left\langle\bar{N}_{\mathcal{T}}(t, z)\right\rangle,[\mathrm{X}] \equiv t / \tau_{p},[\mathrm{Y}] \equiv \varphi(z)\right)$.

sions. This work was supported in part by the Fundamental Metrology Program of the State Committee on Science and Technology.

[1] S. A. Akhmanov, V. A. Vysloukh, A. S. Chirkin, Usp. Fiz. Nauk., 149, No.450 (1986).

[2] S. A. Akhmanov, V. A. Vysloukh, A. S. Chirkin, Optica Femtosekundnyh Lazernykh Impulsov (Optics of Femtosecond Laser Pulses), Moscow, Nauka, 1988 (New York: AIP, 1992).

[3] S. A. Akhmanov, A. V. Belinski, A. S. Chirkin, in Novye Fizicheskie Printsipy Opticeskoi Obrabotki Informatsii (New Optical Methods of Optical Information Processing). (Eds S. A. Akhmanov, M. A. Vorontsov) (Moscow, Nauka 1990) pgs. 83-194.

[4] D. F. Walls, G. J. Milburn, Quantum optics, (Berlin: Springer, 1995).

[5] F. Popescu, A. S. Chirkin, Pisma Zh. Eksp. Teor. Fiz., 69, 482 (1992), [JETP Lett., 69, 516 (1999)].

[6] F. Popescu, A. S. Chirkin, quant-ph/ 0003028/ 8.03.2000/ Submitted to Phys. Rev. A.

[7] S. A. Akhmanov, Yu. E. Dyakov, A. S. Chirkin, Vvedenie v Statisticheskuyu Radiofiziku i Opticu (Introduction to Statistical Radiophysics and Optics) (Moscow: Nauka 1981).

[8] S. A. Akhmanov, A. V. Belinski, A. S. Chirkin, Kbantovaya Electron. (Moscow) 15, 873 (1998), [Quantum Electron. 18, 560 (1988)].

[9] F. König, S. Spälter, I. L. Shumay, A. Sizmann, Th. Fauster, G. Leuchs, J. Mod. Opt., 14, 2425 (1998). 